\title{
Utilización de Cáscara de Arroz como Fuente de Sílice para la Síntesis de Materiales Mesoporosos y su Aplicación en la Remoción de Diferentes Compuestos Orgánicos.
}

\section{Use of rice husk as silica source for the synthesis of mesoporous materials and their application in the degradation of different organic compound.}

\section{Tamara B. Benzaquén}

Centro de Investigación y Tecnología Química (CITeQ - CONICET - UTN) (Universidad Tecnológica Nacional, Facultad Regional Córdoba) - Argentina

tbenzaquen@frc.utn.edu.ar

\section{Paola. M. Carraro}

Centro de Investigación y Tecnología Química (CITeQ - CONICET - UTN) (Universidad Tecnológica Nacional, Facultad Regional Córdoba) - Argentina pcarraro@frc.utn.edu.ar

\section{Griselda. A. Eimer}

Centro de Investigación y Tecnología Química (CITeQ - CONICET - UTN) (Universidad Tecnológica Nacional, Facultad Regional Córdoba) - Argentina

geimer@frc.utn.edu.ar

\section{Resumen}

En el presente trabajo se sintetizaron materiales mesoporosos del tipo MCM-41 utilizando cáscara de arroz como precursor de sílice. En una primera etapa, se evaluaron dos lavados previos de la cáscara de arroz, con agua y ácido nítrico, obteniéndose sílice de alta área superficial para el lavado con ácido. El material mesoporoso silíceo se sintetizó mediante 
el método de tratamiento hidrotérmico utilizando el silicato extraído de la cáscara como precursor de sílice y bromuro de cetiltrimetilamonio (CTAB) como plantilla. El soporte MCM-41 luego fue modificado con Fe por el método de impregnación húmeda para su utilización como catalizadores heterogéneos, en la degradación de diferentes compuestos orgánicos en medio acuoso, como herbicidas (atrazina), compuestos derivados de la industria del plástico (bisfenol A) y de la industria farmacéutica (paracetamol), aplicando la reacción foto-Fenton heterogénea (metal $/ \mathrm{H}_{2} \mathrm{O}_{2} /$ Radiación); y así alcanzar un sistema catalítico de mayor eficiencia.

Palabras claves: cáscara de arroz, MCM-41, PAO's

\begin{abstract}
In the present work, MCM-41-type mesoporous materials were synthesized using rice husk as precursor of silica. In a first stage, two previous washes of the rice husk were evaluated with water and nitric acid, obtaining silica with a high surface area for washing with acid. The siliceous mesoporous material was synthesized by the hydrothermal treatment method using the silicate supernatant extracted from RHA as silica precursor and cetyltrimethylammonium bromide (CTAB) as template. The MCM-41 support was then modified with Fe by the wet impregnation method for its use as heterogeneous catalysts, in the degradation of different organic compounds in aqueous medium, as herbicides (atrazine), compounds derived from the plastic industry (bisphenol A) and the pharmaceutical industry (paracetamol), applying the photo-Fenton heterogeneous reaction (metal $/ \mathrm{H}_{2} \mathrm{O}_{2} /$ Radiation); and thus achieve a catalytic system of greater efficiency.
\end{abstract}

keywords: rice husk, MCM-41, AOPs

\title{
INTRODUCCIÓN
}

Actualmente, los problemas ambientales han promovido el diseño de nuevos materiales más eficientes y amigables con el medio ambiente para ser utilizados en diversas aplicaciones. Los materiales mesoporosos ordenados, como el soporte MCM-41, son de gran interés debido a su estructura bien ordenada, gran superficie específica y su capacidad para interactuar con átomos, iones y moléculas no sólo en la superficie sino también en el interior del material (Carraro et al. 2016, Feng et al. 2019). En particular, sus características específicas los hacen sumamente aptos para su uso en la degradación de contaminantes ya que pueden permitir una buena dispersión de la fase activa, así como un fácil acceso de moléculas voluminosas a los sitios activos en el interior de los poros (Benzaquén et al. 2017). Los principales precursores de sílice utilizados en la síntesis de estos materiales son los alcoxisilanos, como el tetrametoxisilano (TMOS) y tetraetoxisilano (TEOS). En la búsqueda de un precursor más seguro, menos costoso y más respetuoso con el medio ambiente, aparecen las sílices naturales, especialmente las que se encuentran en los residuos agrícolas, que pueden proporcionar una fuente alternativa para reemplazar los precursores comerciales de sílice. Entre ellos, la cáscara de arroz es uno de los desechos agrícolas más comunes con una producción global de 600 millones de toneladas por año de arroz, que genera más de 100 millones de toneladas de cáscaras (Álvarez et al. 2014). Los principales componentes de 
la cáscara de arroz son la celulosa (38\%), hemicelulosas (18\%), lignina (22\%) y SiO2. Este desecho contiene altas cantidades de dióxido de silicio que, a su vez, puede ser una fuente útil de silicio.

Por otro lado, en los últimos años, los Procesos Avanzados de Oxidación (PAO’s) han sido ampliamente utilizados para la destrucción y la mineralización de contaminantes orgánicos no biodegradables en las aguas residuales. Diferentes investigaciones revelan que las estructuras mesoporosas resultan interesantes para ser aplicadas para la degradación agroquímicos (Benzaquén et al. 2018).

Así, en este trabajo se sintetizaron materiales mesoporosos del tipo MCM-41 utilizando cáscara de arroz como precursor de sílice. En una primera etapa, se evaluaron dos lavados previos de la cáscara de arroz, con agua y ácido nítrico, obteniéndose sílice de alta área superficial para el lavado con ácido. El material mesoporoso silíceo se sintetizó mediante el método de tratamiento hidrotérmico utilizando el silicato extraído de la cáscara como precursor de sílice y bromuro de cetiltrimetilamonio (CTAB) como plantilla. El soporte MCM-41 luego fue modificado con Fe por el método de impregnación húmeda para su utilización como catalizadores heterogéneos, en la degradación de diferentes compuestos orgánicos atrazina (ATZ), paracetamol (PCT) y Bisfenol A (BPA) en medio acuoso, aplicando la reacción foto-Fenton heterogénea (metal/ $\mathrm{H}_{2} \mathrm{O}_{2} /$ Radiación); y así alcanzar un sistema catalítico de mayor eficiencia.

\section{Materiales y métodos}

\section{Preparación de la sílice}

La cáscara de arroz proporcionada por una empresa local fue lavada varias veces con agua destilada para remover la suciedad e impurezas adheridas. Luego se secó a $100{ }^{\circ} \mathrm{C}$ y se evaluaron dos métodos de lavados, con agua y ácido nítrico. Una parte fue tratada con una solución de $\mathrm{HNO}_{3}(65 \%$, Cicarelli) $1 \mathrm{M}$ a temperatura ambiente para remover impurezas metálicas. Posteriormente se lavó varias veces y filtró con agua destilada hasta $\mathrm{pH} 7$, y se secó a $100{ }^{\circ} \mathrm{C}$ por $24 \mathrm{~h}$. La cáscara obtenida fue calcinada a $800{ }^{\circ} \mathrm{C}$ por $6 \mathrm{~h}$. El material obtenido en forma de agujas fue mortereado y usado para la extracción de la sílice. El polvo resultante fue mezclado bajo agitación con una solución de $\mathrm{NaOH}$ (Biopack) $1 \mathrm{M}$ a $80^{\circ} \mathrm{C}$. Finalmente, se filtró para separar el sobrenadante. La sílice obtenida fue nombrada $\mathrm{RHA}-\mathrm{SiO}_{2}$.

\section{Síntesis de MCM-41-RHA}

Los silicatos mesoporosos del tipo MCM-41 se sintetizaron por el método de tratamiento hidrotérmico utilizando el precursor de sílice obtenido anteriormente y bromuro de cetil trimetil amonio (CTAB, Merck) como agente moldeante. En primer lugar, el CTAB se agregó a la solución de $\mathrm{NaOH}$ a $40^{\circ} \mathrm{C}$. Luego, se adicionó la fuente de silicio, agitando vigorosamente por $7 \mathrm{~h}$ a temperatura ambiente y luego se llevó a estufa durante $24 \mathrm{~h}$ a $100^{\circ} \mathrm{C}$. El sólido resultante se filtró, lavó con agua destilada hasta alcanzar $\mathrm{pH} 7$ y secó a $60^{\circ} \mathrm{C}$ en estufa durante $12 \mathrm{~h}$. Finalmente, el agente plantilla se evacuó de las muestras mediante calentamiento bajo flujo de $\mathrm{N} 2$ hasta $500{ }^{\circ} \mathrm{C}$ durante $6 \mathrm{~h}$ y posterior calcinación a $500{ }^{\circ} \mathrm{C}$ durante $6 \mathrm{~h}$ bajo flujo de aire.

El soporte sintetizado MCM-RHA se modificó con 2,5, 5 y $10 \%$ p/p de Fe por el método de impregnación húmeda, utilizando una solución de $\mathrm{Fe}\left(\mathrm{NO}_{3}\right)_{3} \cdot 9 \mathrm{H}_{2} \mathrm{O}$ (Merck a.c.s.) con la concentración correspondiente a la carga de Fe deseada (\%p/p). Posteriormente se removió el agua en un evaporador rotatorio y el sólido final se secó en estufa y calcinó durante $3 \mathrm{~h}$ a 
$350{ }^{\circ} \mathrm{C}$. Los materiales resultantes se nombraron de la siguiente manera: Fe/MCM-RHA(x), donde $\mathrm{x}$ es la concentración inicial de la solución de impregnación en \%p/p.

\section{Caracterización de los catalizadores}

Los patrones de difracción de rayos $\mathrm{X}$ (DRX) se recogieron en un difractómetro Philips $\mathrm{PW}$ 3830 , con radiación $\mathrm{Cu}-\mathrm{Ka}(\lambda=1,5418 \AA$ ). Las propiedades texturales se determinaron utilizando un equipo Micromeritics ASAP 2000. Los espectros Ultravioleta Visible con Reflectancia Difusa (UV-Vis RD) se recolectaron utilizando un espectrómetro Jasco V 650 equipado con esfera integradora. Las imágenes de microscopía electrónica de barrido (SEM) de los materiales se obtuvieron en un equipo FE-SEM $\sum$ igma-ZEISS.

\section{Dispositivo experimental}

El dispositivo experimental donde se evaluó la acción catalítica de los materiales fue un agitador mecánico múltiple compuesto por de 4 reactores de vidrio borosilicato (PARSEC, Aries VII). El mismo se encuentra equipado con 4 paletas recubiertas de teflón, movidas sincrónicamente por un motor de velocidad variable entre 50-250 rpm, permitiendo realizar 4 corridas en simultáneo. Los reactores batch, fueron irradiados desde el fondo por 4 lámparas tubulares UV-vis (Philips Actinic BL TL-K 40W/10-R. Las mismas se encuentran ubicadas paralelamente a una distancia de $5 \mathrm{~cm}$ de la base de cada reactor; y emiten de a pares en las aberturas circulares, en un espectro continuo entre entre 340 y $410 \mathrm{~nm}$, con pico en $365 \mathrm{~nm}$ y tres bandas a 404, 408 y $438 \mathrm{~nm}$. Esto fue verificado mediante mediciones realizadas con un equipo UV-AB Meter (Tenmars TM-213). En cada reactor se colocó una suspensión acuosa del contaminante; ATZ (( $\geq 98 \%$, Sigma-Aldrich), BPA (99\%, Aldrich) y PCT (99\%, Aldrich) en concentraciones de $30 \mathrm{ppm}$; y una distribución del material mesoporoso sintetizado con una concentración de $1 \mathrm{~g} \mathrm{~L}^{-1}$. El dispositivo incluye un sistema de refrigeración, el cual se compone de 4 serpentines de tubo cobre de $7 \mathrm{~mm}$ de diámetro exterior, con espesor de pared de 1 $\mathrm{mm}$, por el cual se hace circular agua refrigerada para mantener el sistema en condiciones isotérmicas $\left(20^{\circ} \mathrm{C}\right)$. Finalmente, cada reactor se encuentra equipado con un dispositivo difusor de aire y una válvula de toma de muestra.

\section{Determinaciones analíticas}

Las muestras de reacción fueron filtradas con filtros de papel (Double Rings, N.203) para recuperar el catalizador y divididas posteriormente en alícuotas para determinaciones de: (i) ATZ, PCT y BPA mediante cromatografía Líquida de Alta Resolución (HPLC) en un cromatógrafo Perkin Elmer (Serie 200), equipado con detector UV y con columna de fase reversa C18 (Agilent); la composición de la fase móvil (flujo de $1 \mathrm{~mL} \mathrm{~min}^{-1}$ ) y la detección empleada dependió de la molécula orgánica a cuantificar: ATZ y BPA, mezcla binaria de agua destilada y metanol (30:70), y para el PCT mezcla binaria de agua destilada y metanol (70:30); con detección a una longitud de onda de 221, 243 y $278 \mathrm{~nm}$, respectivamente; (ii) de peróxido de hidrógeno $\left(\mathrm{H}_{2} \mathrm{O}_{2}\right)$ mediante una técnica iodométrica modificada con determinaciones en un espectrofotómetro, UV-VIS Jasco V-650, a $350 \mathrm{~nm}$ (Allen et al. 1952); y (iii) de $\mathrm{Fe}^{2+}$ y Fe total, en el caso de la reacción foto-Fenton homogénea, empleando el método colorimétrico 1,10-fenantrolina a $510 \mathrm{~nm}$ (Eaton et al. 1995). 


\section{Resultados y discusión}

\section{Caracterización de los materiales}

La tabla 1 muestra las propiedades texturales de los materiales estudiados obtenidas de las isotermas de adsorción-desorción de $\mathrm{N}_{2}$ a $77 \mathrm{~K}$. De los resultados obtenidos se observa como con el lavado con HNO3 se mejoró notablemente la superficie especifica en comparación con el lavado con agua $\left(216 \mathrm{~m}^{2} / \mathrm{g}\right.$ y $27 \mathrm{~m}^{2} / \mathrm{g}$ respectivamente). En la Figura 1.a se muestra la isoterma de la muestra $\mathrm{RHA}-\mathrm{SiO}_{2}$ lavada con ácido nítrico. De acuerdo a la clasificación de la IUPAC (Everett et al. 1971, Thi Thu et al. 2019), este material presentó una isoterma del tipo IV, con un bucle de histéresis del tipo H1. La distribución del tamaño de poro de esta muestra está en el rango de 15-65 Å aproximadamente.

Las isotermas de adsorción - desorción de $\mathrm{N}_{2}$ del soporte MCM-RHA y los catalizadores modificados con Fe se muestran en la Figura 1.b. Todos los sólidos presentaron isotermas de tipo IV, típicas de estructuras mesoporosas de acuerdo con la clasificación de la IUPAC (Everett et al. 1971, Neimark et al. 2001), con un pronunciado aumento de la adsorción en el intervalo de presiones relativas entre 0,2 a 0,35 , característico de una estrecha región de condensación capilar dentro de los mesoporos. Esta característica concuerda con las estrechas distribuciones de tamaños de poro unimodales, observadas en la Figura 1.b (DTP). Sin embargo, mientras que la inflexión es aguda para el sólido MCM-41, dando cuenta de una estrecha distribución de tamaños de poro, al aumentar la carga de Fe se hace menos pronunciada, lo que indica un rango más amplio en la distribución de tamaños de poro (Carraro et al. 2014).

Además, todas las muestras presentaron alta superficie específica $\left(\mathrm{S}_{\mathrm{BET}}\right)$ y volumen de poros $\left(\mathrm{V}_{\mathrm{TP}}\right)$, típicos de materiales mesoporosos. Sin embargo, una ligera disminución de estos valores se observa con el aumento en la carga de hierro. Así, es posible observar en la DTP, un pequeño cambio en el tamaño de poro con el contendido de hierro. Esto se puede atribuir al bloqueo de algunos poros debido al aumento en la presencia de clústers y/o pequeñas partículas de óxidos metálicos finamente dispersos dentro de los canales, como así también grandes partículas en la superficie externa.

\begin{tabular}{|c|c|c|c|}
\hline Catalizador & $S_{B E T}\left(m^{2} / g\right)$ & $\mathrm{Dp}(\AA)$ & $V_{T P}\left(\mathrm{~cm}^{3} / \mathrm{g}\right)$ \\
\hline 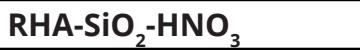 & 216 & - & - \\
\hline RHA-SiO $-\mathrm{H}_{2} \mathrm{O}$ & 27 & - & - \\
\hline MCM-RHA & 1019 & 30 & 0.99 \\
\hline $\mathrm{Fe} / \mathrm{MCM}-\mathrm{RHA}(2.5)$ & 930 & 28 & 0.74 \\
\hline Fe/MCM-RHA(5) & 877 & 29 & 0.66 \\
\hline Fe/MCM-RHA(10) & 843 & 28 & 0.66 \\
\hline
\end{tabular}

Tabla 1. Propiedades texturales de los materiales estudiados 

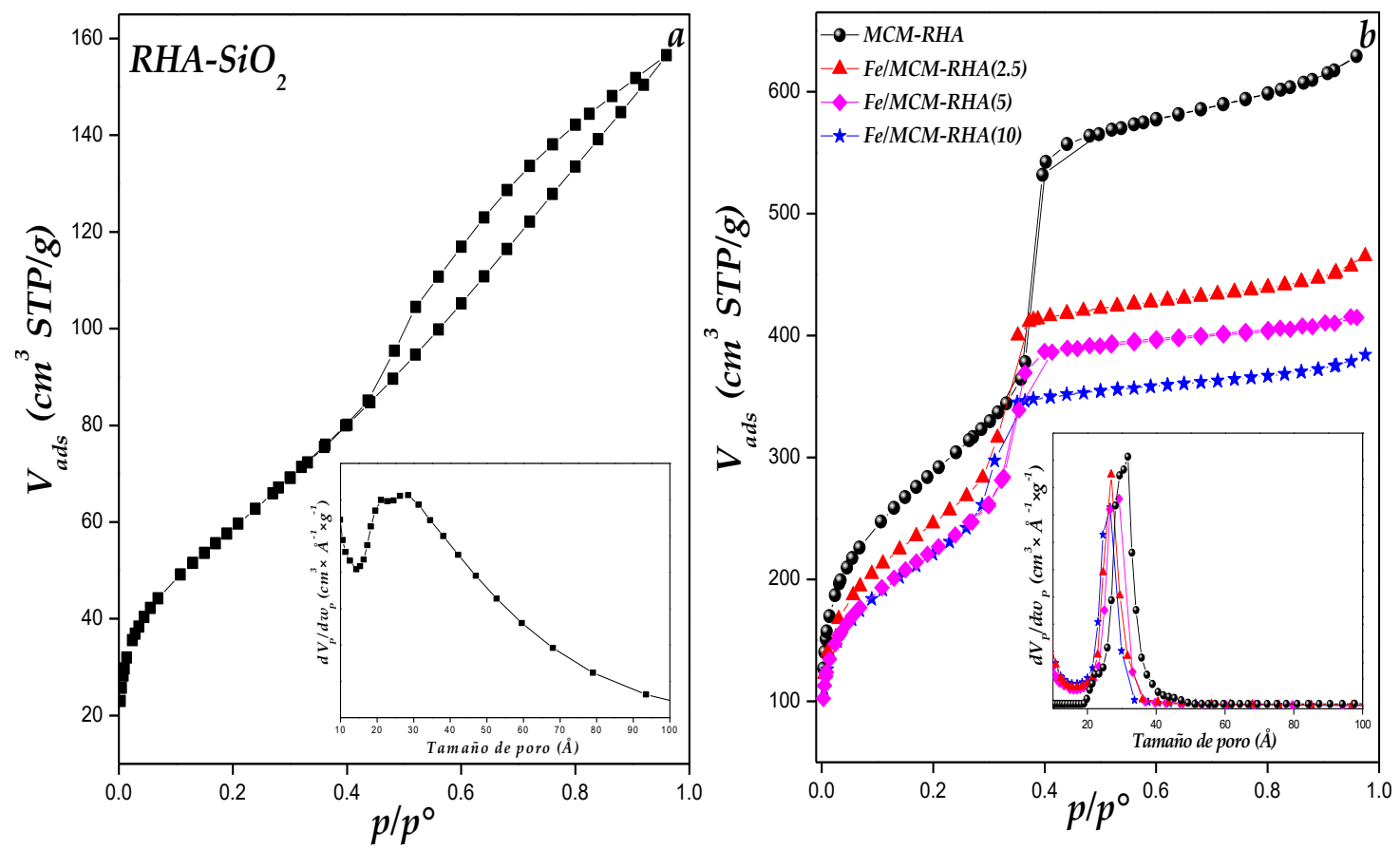

Figura 1. Isotermas de adsorción - desorción de $\mathrm{N}_{2}$ a $77 \mathrm{~K}$ de (a) $\mathrm{RHA}_{-} \mathrm{SiO}_{2} \mathrm{y}$ (b) de los materiales sintetizados. Inset: Distribución de tamaño de poro (DTP).

En la Figura 2 se presentan los espectros UV-vis RD de todas las muestras investigadas. Se pueden observar tres bandas a 220-250, 300-380 and 450-550 nm aproximadamente. El primer máximo a alrededor de $254 \mathrm{~nm}$ indica que los átomos de hierro pueden unirse a los átomos de O de la superficie (Cuello et al. 2015, Benzaquén et al. 2017). Mientras que el segundo máximo a aproximadamente $340 \mathrm{~nm}$ puede atribuirse a grupos de óxido de hierro $(\mathrm{FeO})_{\mathrm{n}}$ o nanopartículas de muy pequeño tamaño (Cuello et al. 2015). Por último, el tercer máximo a aproximadamente $500 \mathrm{~nm}$ puede asignarse a nanopartículas de óxido de hierro de mayor tamaño (Liu et al. 2004). Es conocido que la posición del borde de absorción de polvos semiconductores es afectada significativamente por el tamaño de partícula, desplazándose a menores longitudes de onda con la disminución del tamaño de las mismas. Así, se puede observar un corrimiento de estas bandas a mayores longitudes de onda en las muestras con altas cargas de hierro, debido al efecto cuántico de tamaño de partícula, lo que indica una mayor presencia de nanopartículas de óxido de hierro más grandes. 


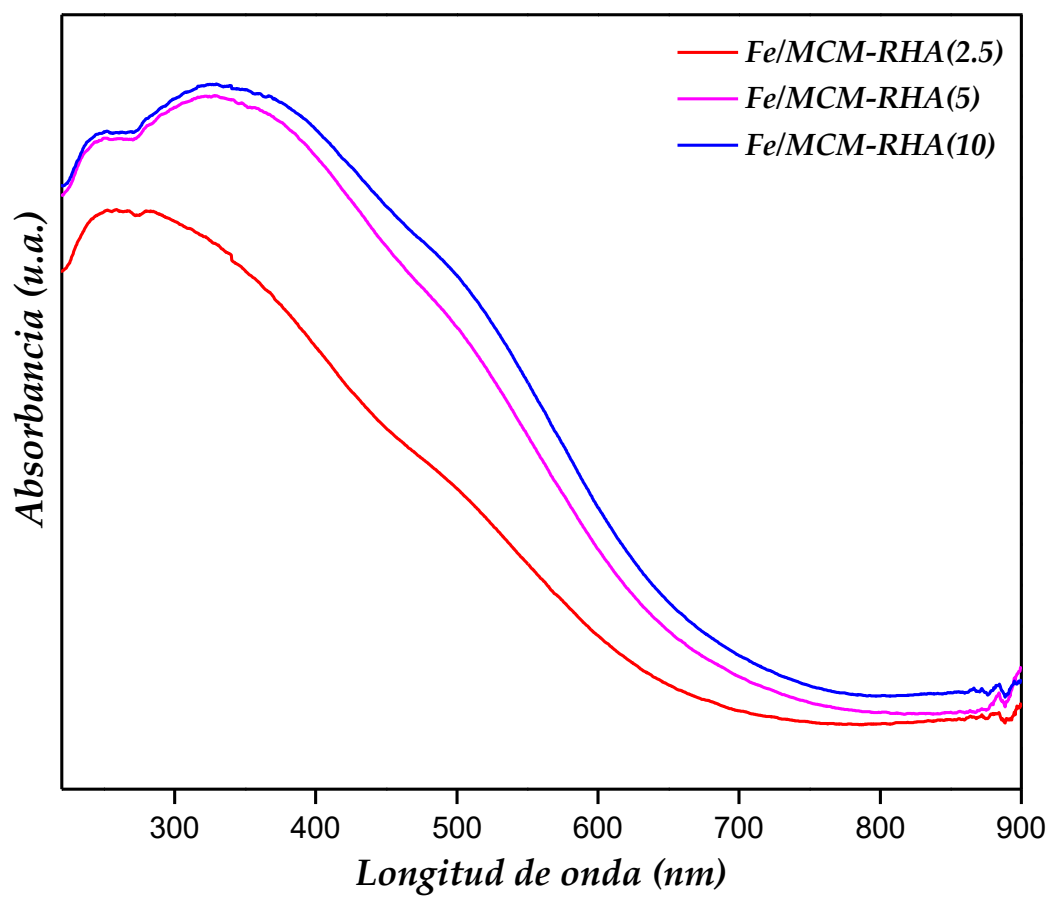

Figura 2. Espectro de UV-Vis RD de los materiales modificados con Fe

La morfología y el tamaño de partícula de los materiales mesoporosos se investigaron por microscopía de barrido electrónico (SEM). Las micrografías SEM de algunas muestran tomadas como representativas se muestran en la Figura 3. Se observan diferencias significativas en las imágenes del material lavado con agua y ácido nítrico. Éste último (Fig.3 (a)) presenta una morfología esférica. Luego, las imágenes de los materiales sintetizados (Fig.3 (c) y (d)) indican que las partículas no poseen una morfología particular, aunque se observan pequeñas partículas con forma esférica, las cuales se segregan formando aglomerados en un amplio rango de tamaños (Thi Thu et al. 2019). A pesar de que estas muestras poseen una estructura mesoporosa altamente ordenada, las partículas no presentan una morfología bien definida y existe una agregación de partículas de tamaños irregulares. 

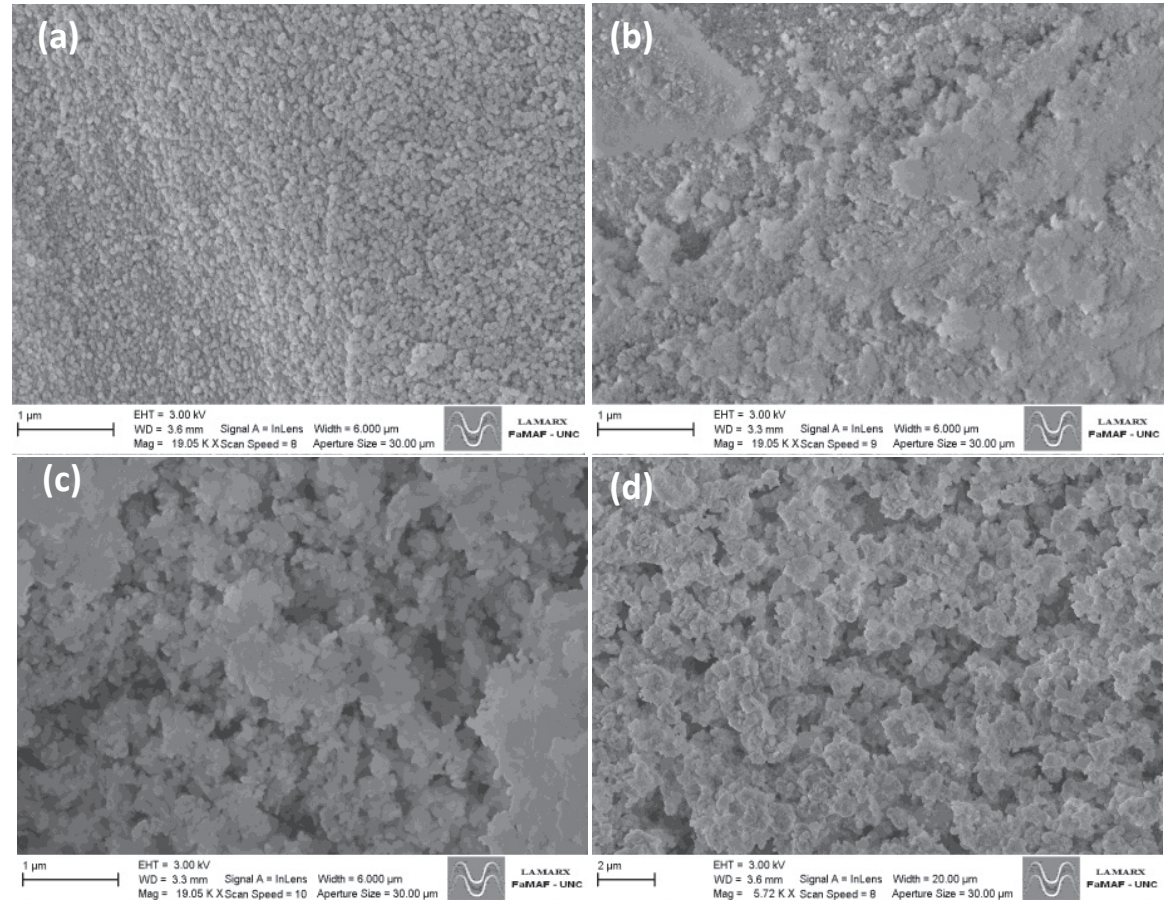

Figura 3. Imágenes SEM (a) $\mathrm{SiO}_{2}-\mathrm{RHA}^{-}-\mathrm{HNO}_{3^{\prime}}$ (b) $\mathrm{SiO}_{2}-\mathrm{RHA}-\mathrm{H}_{2} \mathrm{O}$, (c) del soporte MCM-RHA y (d) de Fe/MCM-RHA(5).

\section{Evaluación catalítica de los catalizadores}

Se investigó la viabilidad de degradación de diferentes contaminantes, aplicando la reacción tipo foto-Fenton Heterogénea con diferentes cargas de Fe sobre el soporte mesoporoso ordenado de sílice MCM-41, bajo las mejores condiciones de reacción previamente establecidas en trabajos anteriores (Benzaquén et al. 2017, Carraro et al. 2018).

La Figura 4 muestra la degradación de ATZ, PCT y BPA en función del tiempo para los diferentes catalizadores sintetizados, utilizando radiación UV-visible, temperatura ambiente, un $\mathrm{pH}$ cercano al neutro y manteniendo constante la concentración inicial de catalizador $\left(\mathrm{C}_{\mathrm{CAT}}^{0}\right)$, la concentración inicial de peróxido de hidrógeno $\left(\mathrm{C}_{\mathrm{H} 2 \mathrm{O} 2}^{0}\right)$ y la radiación. Como se observa en estas figuras, los catalizadores con las mayores cargas de metal mostraron una disminución importante en la actividad catalítica para todos los contaminantes evaluados. Cuando el contenido de Fe fue del $2.5 \%$ p/p (Fe/MCM-RHA(2.5)), la degradación de todos los contaminantes alcanzó su valor máximo. En base a estos resultados, como se puede observar, el bisfenol A fue el contaminante que alcanzó el mayor grado de degradación.

Como se ha señalado anteriormente, las muestras con altas cargas de hierro presentaron una mayor presencia de nanopartículas de óxido de hierro de mayor tamaño. Dichas especies, podrían estar bloqueando algunos sitios responsables de la actividad de los catalizadores. Así, en la muestra con menor contenido de Fe, las especies de activas de metal estarían finamente dispersas en la estructura de sílice y activas para la fotodegradación. 

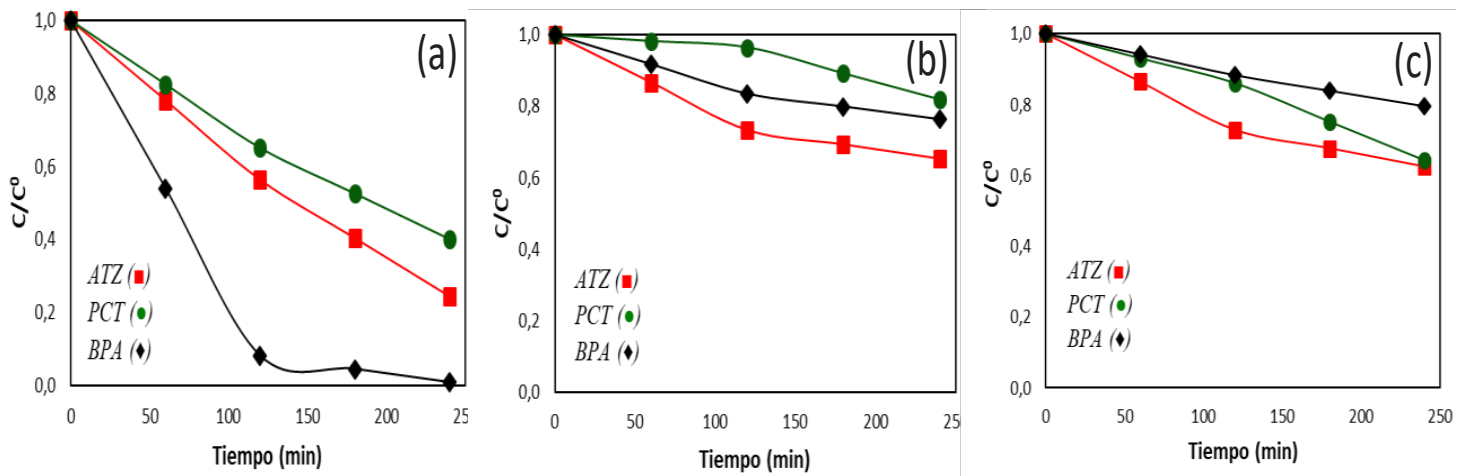

Figura. 4. Concentración relativa de contaminantes en función del tiempo para el proceso fotoFenton heterogéneo: para (a) Fe/MCM-RHA(2.5), (b) Fe/MCM-RHA(5) y (c) Fe/MCM-RHA(10).

Por último, previo a cada reacción se buscó alcanzar el equilibrio de adsorción de contaminante sobre el material. Esto se llevó a cabo poniendo en contacto la cantidad deseada de catalizador con la solución de contaminante bajo agitación, flujo de aire y oscuridad, por un período de $60 \mathrm{~min}$. En todos los casos el porcentaje de adsorción se mantuvo alrededor del 10-20\%, dependiendo del material y del contaminante evaluado. Cabe mencionar que el BPA fue el compuesto que presentó los menores porcentajes de adsorción para los tres materiales evaluados.

Finalmente, en todos los casos se estudió el posible "leaching" de metal, luego de finalizada la reacción. Los resultados obtenidos arrojaron valores menores a $0,8 \mathrm{ppm}$, por lo que, los materiales presentarían una considerable estabilidad.

\section{Conclusiones}

Los silicatos mesoporosos MCM-41 fueron sintetizados utilizando cáscara de arroz como precursor de sílice y modificados con diferentes contenidos de Fe por el método de impregnación húmeda. Todos los materiales exhibieron alta superficie específica, volumen de poros y buena regularidad estructural que retiene la estructura característica de materiales mesoporoso luego de la incorporación del metal.

Estos materiales mesoestructurados se han probado con éxito para determinar la degradación foto-Fenton heterogénea de diferentes contaminantes orgánicos. El catalizador con la menor carga de hierro $(2.5 \% \mathrm{p} / \mathrm{p}$ de $\mathrm{Fe})$ presentó la mayor degradación de contaminantes, probablemente debido a presencia de especies activas de Fe, altamente dispersas e interactuando con la superficie de soporte.

\section{Agradecimientos}

Los autores agradecen a la Universidad Tecnológica Nacional (MAUTICO0004427TC), al Ministerio de Ciencia y Tecnología de la Provincia de Córdoba (PGC Nº 000045/2016) y Agencia Nacional de Promoción Científica y Tecnológica (PICT-2015 1485) por el apoyo financiero. 


\section{Referencias}

Allen A.O., Hochanadel J.A., Ghormley J.A., Davis T.W. (1952). "Decomposition of Water and Aqueous Solutions under Mixed Fast Neutron and $\gamma$-Radiation”, J. Phys. Chem. 56, 575.

Álvarez J., López G., Amutio M., Bilbao J., Olazar M. (2014). "Upgrading the rice husk char obtained by flash pyrolysis for the production of amorphous silica and high quality activated carbon", Bioresource Technology 170, 132.

Benzaquén T.B., Cuello N.I., Alfano O.M., Eimer G.A. (2017). "Degradation of Atrazine over a heterogeneous photo-fenton process with iron modified MCM-41 materials", Catalysis Today 296, 51.

Benzaquén T., Barrera D., Carraro P., Sapag K., Alfano O., Eimer G. (2018).

"Nanostructured catalysts applied to degrade atrazine in aqueous phase by heterogeneous photo-Fenton process”, Env. Sc. Pol. Res. 26, 4192.

Carraro P.M., Elías V.R., García Blanco A.A., Sapag K., Moreno S., Oliva M.I., Eimer G.A. (2014). "Synthesis and multi-technique characterization of nickel loaded MCM-41 as potential hydrogen-storage materials”, Micropor. Mesopor. Mater. 191, 103.

Carraro P. M., García Blanco A. A., Soria F. A, Lener G., Sapag K., Eimer G. A., Oliva M. I. (2016). "Understanding the role of nickel on the hydrogen storage capacity of Ni/MCM-41 materials", Journal of Microporous and Mesoporous Materials 23,131.

Carraro P.M., Benzaquén T.B., Alfano O.M., Oliva M.I, Eimer G.A. (2018). “Advanced oxidation technologies to remove endocrine disruptors in water effluents based on zinc supported mesoporous catalyst”, Revista Mexicana de Ing. Qca 13(3), 1135.

Cuello N.I., Elías V.R., Rodriguez Torres C.E., Crivello M.E., Oliva M.I., Eimer G.A. (2015). "Development of iron modified MCM-41 as promising nano-composites with specific magnetic behavior”, Micropor. Mesopor. Mater.203, 106.

Eaton A.D., Clesceri L.S., Rice E.W., Greenberg A.E. (1995). APHA AWWA WEF Standard Methods for the examination of Water and Waste Water, 19th Ed.

Feng D., Feng Y., Qiu L., Li P., Zang Y., Zou H., Yu Z., Zhang X. (2019). “Review on nanoporous composite phase change materials: Fabrication, characterization, enhancement and molecular simulation", Renewable and Sustainable Energy Reviews 109, 578.

Everett D.H. (1971). Manual of symbols and terminology for physicochemical quantities and units-Appendix II. Definitions, terminology and symbols in colloid and surface chemistry. Part I. IUPAC.

Liu S., Wang Q., Van Der Voort P., Cool P., Vansant E., Jiang M. (2004). “Magnetism of ironcontaining MCM-41 spheres”, J. Magn. Mater. 280, 31.

Neimark A.V., Ravikovitch P.I. (2001). "Capillary condensation in MMS and pore structure characterization”, Micropor. Mesopor. Mat. 44-45, 697.

Thi Thu H., Tien Dat L., Anh Tuan V. (2019). "Synthesis of mesoporous SiO2 from rice husk for removal of organic dyes in aqueous solution”, Vietnam J. Chem. 57(2), 175. 